\title{
Preservation of sural nerve in classic forms of Guillain-Barré in a Mexican health institution
}

\author{
Juan José Gómez-Piña ${ }^{1 *}$, Christopher Cabib ${ }^{1}$, Bruno Estañol $^{2}$, and Erwin Chiquete ${ }^{1}$ \\ ${ }^{1}$ Department of Neurology and Psychiatry; ${ }^{2}$ Department of Neurophysiology, Instituto Nacional de Ciencias Médicas y Nutrición Salvador Zubiran, \\ Mexico City, Mexico
}

\begin{abstract}
Background: The term sural sparing (SS) consists of the early finding in the nerve conduction studies (NCS) of patients with Guillain-Barré syndrome (GBS) of the preservation or normality of the sural nerve with abnormality in sensory nerves of thoracic limbs. Its pathophysiology lies in the greater vulnerability to demyelinating sensory damage in distal segments of the hand than proximally in the calf. The SS is highly specific of acute inflammatory demyelinating polyradiculoneuropathy $(A I D P)$ and is occasionally found in acute motor/sensory axonal neuropathy (AMAN/AMSAN). Objective: We aim to describe the prevalence of SS among the forms of GBS in patients hospitalized in our institute. Materials and methods: We reviewed 61 cases of confirmed GBS (19 demyelinating, 25 axonal, and 17 unclassified forms) corresponding to the 1999-2017 period. Exclusion criteria were as follows: NCS report not available or performed 21 days after the onset of symptoms, chemotherapy in the past 2 years, and/or previous polyneuropathy. SS was defined as the preserved amplitude in sensory action potentials (SAPs) of the sural nerve with abnormal findings in median and/or ulnar nerve SAPs. Results: Thirty patients (21 men, mean $45.5 \pm 21.2$ years) met the selection criteria, distributed in 12 AIDP, 3 Miller-Fisher syndromes, 9 AMAN, and 6 AMSAN. The NCS was performed $9.1 \pm 6.0$ days from debut. There were no significant differences in demographic variables or in the amplitude of SAPs between demyelinating and axonal forms. Two patients with AIDP presented SS (16.7\%), which was not observed in any other form of GBS. Conclusion: We conclude that, despite the high specificity of SS for AIDP, its low prevalence and the high prevalence of axonal forms in Mexican population suggest that SS is not a suitable electrophysiological screening parameter for differentiating forms of GBS.
\end{abstract}

Key words: Sural nerve. Guillain-Barré. Sural sparing. Acute inflammatory demyelinating polyradiculoneuropathy.

\section{Preservación del nervio sural en formas clasicas de Guillain-Barré en una institución de salud en México}

\section{Resumen}

Antecedentes: La preservación sural (PS) es muy específica de la polirradiculoneuropatía desmielinizante inflamatoria aguda (PDIA) y se encuentra de modo ocasional en la neuropatía axonal motora/sensorial aguda (NAM/NASA). Objetivo: Describir la prevalencia de PS entre las formas del síndrome de Guillain-Barré (SGB) en pacientes hospitalizados en la institución

Correspondece:

*Juan José Gómez-Piña

E-mail: drjgomezp@gmail.com
Available online: 11-02-2021 Rev Mex Neuroci. 2021;22(1):10-14 www.revmexneurociencia.com 1665-5044/ @ 2020 Academia Mexicana de Neurología A.C. Published by Permanyer. This is an open access article under the CC BY-NC-ND license (http://creativecommons.org/licenses/by-nc-nd/4.0/). 
de los autores. Material y métodos: Se revisaron 61 casos de SGB confirmados (19 formas desmielinizantes, 25 axonales y 17 no clasificadas) correspondientes al período 1999-2017. Resultados: No se observaron diferencias significativas en las variables demográficas o la amplitud de los potenciales de acción sensorial (PAS) entre las formas desmielinizantes y axonales. Dos pacientes con PDIA presentaron PS (16.7\%), que no se observó en ninguna otra forma del SGB. Conclusión: A pesar de la alta especificidad de PS para PDIA, su baja prevalencia y la elevada prevalencia de formas axonales en la población mexicana sugieren que la PS no es un parámetro de detección electrofisiológico adecuado para diferenciar las modalidades del SGB.

Palabras clave: Nervio sural. Síndrome de Guillain-Barré. Preservación sural. PDIA.

\section{Introduction}

Guillain-Barré syndrome (GBS) is classically presented by acute areflexic tetraparesis, which is potentially fatal because it compromises respiratory musculature and it is associated with autonomic dysfunction. The pathophysiology of GBS lies in the immune damage caused by autoantibodies production against myelin, and the axonal membrane of spinal roots and peripheral nerves, which, depending on the predominant pathophysiologic mechanism, classically cause two types of abnormalities: slowing of conduction speed and nerve conduction blockages, when primary demyelination occurs, or Wallerian degeneration, in relation to primary axonal damage 1 .

The neurophysiological diagnosis of GBS includes the application of criteria, aimed at demonstrating phenomena of primary demyelination in motor nerves, such as delayed distal latencies, slowing of conduction, and conduction blocks ${ }^{2,3}$

The presence of some of these criteria, and the typical clinical picture, supports the diagnosis of GBS. For axonal forms, it is considered a diagnosis that does not meet criteria for primary demyelination and that the clinical picture is compatible. However, it has recently been shown that even in the axonal variants, antibodies directed against the components of the node and paranodal region can also generate conduction blocks, at axonal level ${ }^{4}$. However, alterations in sensory nerves are not commonly part of the established diagnostic criteria, despite the fact that sensory symptoms are prevalent in GBS.

The spinal roots and terminal segments of peripheral nerves are anatomical sites susceptible to autoimmune damage in GBS 5 .

In fact, it is not unusual to find in nerve conduction studies (NCSs) performed early during the evolution of the clinical picture of GBS, abnormality in sensory nerves in upper extremities with normality conduction in the lower extremities, a phenomenon commonly known as "sural nerve preservation" (SS, from the
English sural sparing) ${ }^{3}$. It is possible explanation lies in two technical aspects related to the pathophysiology of GBS: first, the recording of the sensory action potentials (SAPs) of the sural nerve is performed in segments that are not as distal, as it is done in the upper extremities, where they are less susceptible to demyelinating damage and, second, in the longer time required in longer sensory nerves (for example, sural nerve) to observe reduction in the amplitude of the SAP when Wallerian degeneration occurs due to axonal damage in the spinal roots $^{5}$.

Although the sensitivity reported for SS as a marker of GBS is low $(20 \%)$, its presence is usually considered to be highly specific for acute inflammatory demyelinating polyradiculoneuropathy (AIDP) ${ }^{6}$, although in axonal forms, cases have been reported exceptionally. The present study aims to describe the prevalence of SS among the classic forms of GBS in patients hospitalized in our institute.

\section{Materials and methods}

We retrospectively reviewed a total of 103 clinical files with diagnosis of presumptive discharge of GBS corresponding to the period 1999-2017 of the National Institute of Medical Sciences and Nutrition Salvador Zubirán (INCMNSZ), evaluating the presence of Hadden criteria for SGB, as well as the clinical presentation, such as the presence of neuropathy associated with another disease, data of carpal tunnel syndrome, dysesthesia, or dysautonomia, which preliminarily excluded 42 of these patients, in whom GBS was ruled out, or had serological alterations, that is, they presented uncontrolled diabetes mellitus, storage diseases, electrolyte alterations, use of neurotoxic drugs, and/or an alternative diagnosis was found.

On the other hand, a total of 61 patients with the diagnosis of GBS were included, according to the clinical picture, evolution, and the neurophysiological criteria. 
Table 1. Electrophysiological values found in our patients, and proposed by Hadden classification, in brackets

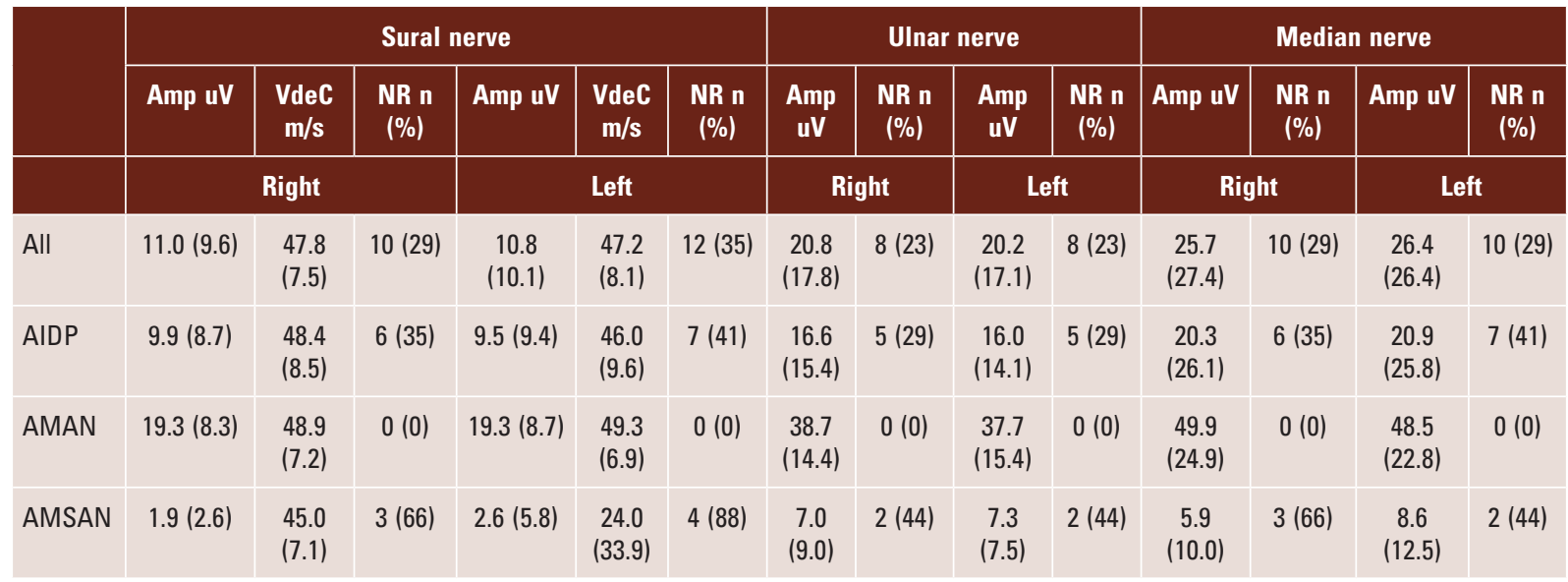

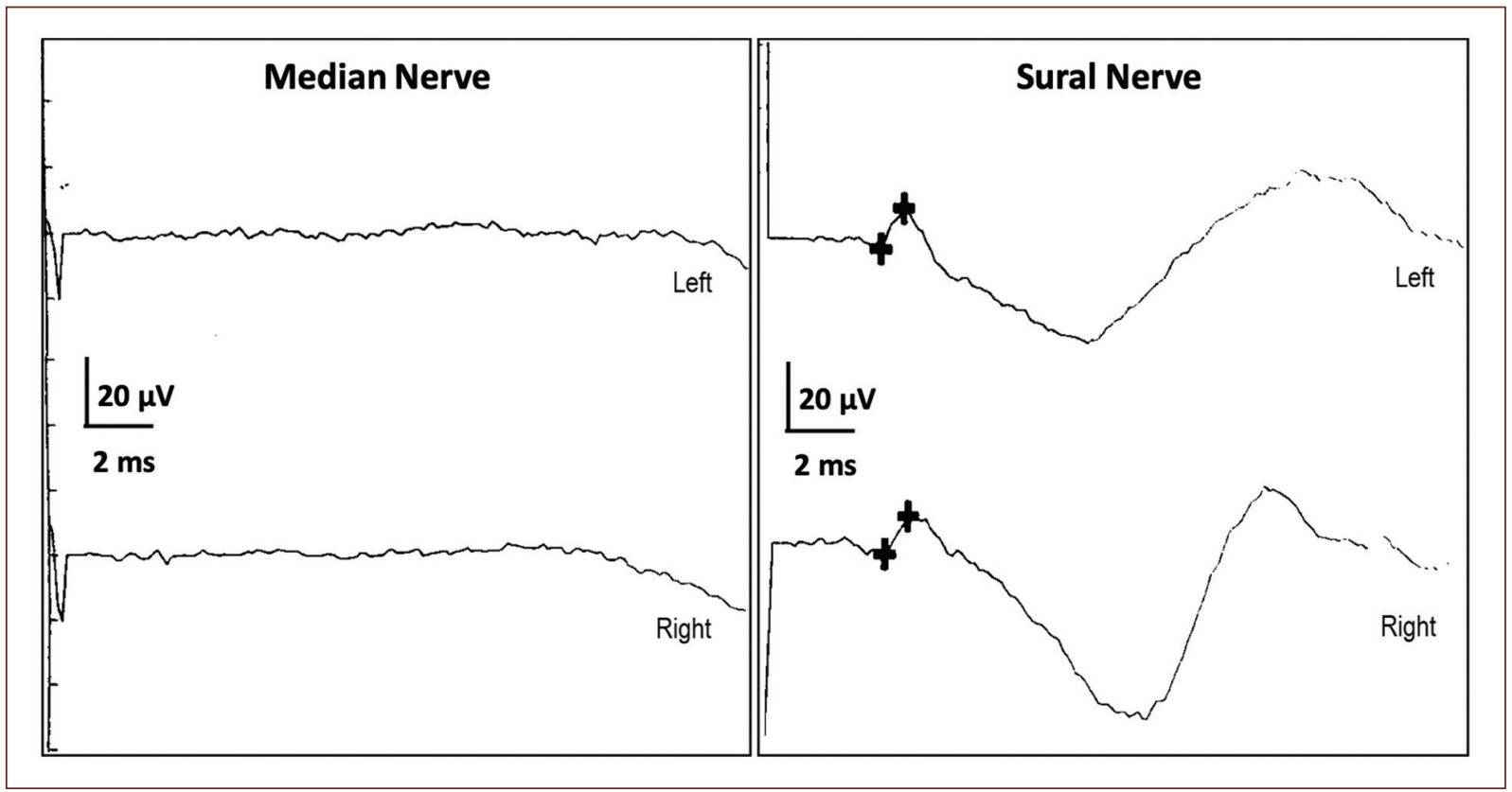

Figure 1. Presentation of the sural sparing phenomenon in the sural nerve, compared to normal median nerve.

Once the true cases of GBS were identified, we identified each of them according to the clinical presentation subtype, differentiating them from demyelinating (AIDP), axonal, pure motor (acute motor axonal neuropathy [AMAN]) or sensory motor (acute sensory axonal neuropathy [AMSAN]) forms, as well as Miller-Fisher (MF) variant. Subsequently, we analyzed the electrophysiological variants such as amplitude and speed of conduction of sural, ulnar, and median nerves, establishing values of normality according to the criteria of Hadden and Rajabally (Table 1).

SS was defined as normality in the amplitude or relative preservation of the sensory nerve action potential (SNAP) of the sural nerve with abnormality of the
SNAPs of the median and/or ulnar nerve ${ }^{5}$, excluding from the final analysis, the cases according to previously defined criteria ${ }^{1}$ (Fig. 1).

We performed the statistical analysis with the statistical package SPSS 20 , considering statistically significant differences with $p<0.05$.

\section{Results}

Thirty-four patients met the selection criteria for SGB, which presented the following characteristics. Neurophysiological parameters showed a lower amplitude of the PANS and a high frequency of NR in AIDP and AMSAN compared to AMAN (Table 2). 
Table 2. Relationship between the clinical presentations of GBS and the variables of age, sex, time of evolution, and presence of diabetes

\begin{tabular}{|l|c|c|c|c|}
\hline & All & AIDP & AMAN & AMSAN \\
\hline Age (years) & $44.2 \pm 20.0$ & $48.5 \pm 21.8$ & $35.8 \pm 19.0$ & $42.0 \pm 10.1$ \\
\hline $\begin{array}{l}\text { Gender } \\
\text { (n, female, (\%)) }\end{array}$ & $13(28)$ & $7(41)$ & $4(44)$ & $1(20)$ \\
\hline $\begin{array}{l}\text { Time evolution } \\
\text { (days) }\end{array}$ & $10.4 \pm 6.1$ & $10.5 \pm 6.5$ & $8.9 \pm 6.5$ & $10.6 \pm 5.0$ \\
\hline $\begin{array}{l}\text { Diabetes } \\
\text { (n, (\%)) }\end{array}$ & $4(10)$ & $2(5)$ & $0(0)$ & $1(2)$ \\
\hline
\end{tabular}

Seven patients with AIDP showed SS (17.6\%) (which was not observed in the other forms of GBS), two of them corresponding to "extreme" SS (absent median nerve PANSs).

\section{Discussion}

Our study has the limitation that it is a retrospective study, in addition to using only the Hadden criteria for the diagnosis of GBS. Historically, neurophysiological criteria for the diagnosis of GBS are applied to motor nerves and not to sensory nerves. This is partly because the demonstration of the phenomena of primary demyelination in sensory nerves is technically more difficult, for example, mainly related to the high degrees of temporal dispersion of their potentials when stimulated in more than 1 point. However, some authors consider SS to be a useful tool in the diagnostic support of acquired demyelinating polyneuropathies, including GBS and chronic inflammatory demyelinating polyneuropathy ${ }^{5}$.

The previous studies have shown that SS has a sensitivity of $38 \%$ in the diagnosis of AIDP, with a specificity of $90.9 \%$ using the Hadden criteria, while a sensitivity of $36.8 \%$ and specificity of $69.6 \%$ have been found for AIDP with the Rajabally's criteria?. It is necessary that the neurophysiological study be performed with some precocity during the evolution of the disease (typically before 21 days from the onset of symptoms), since in later stages, phenomena of secondary axonal degeneration of the sural nerve can obscure its presence.

On the other hand, the terminology "sural nerve preservation" (SS) in GBS depends on the definition that is used, for example, that normality is considered versus the preservation of the PANS of the sural or of the sensory nerves that are explored in upper extremities (median, ulnar, or radial nerve) to be confronted with the sural. Classically, the SS seems to be specific of the demyelinating forms that imply the absence of a median nerve response, independently of the criteria used for the classification of the GBS subtype. However, some authors consider that the use of the median nerve can increase the false positives of SS for GBS considering the high prevalence of compression of the median nerve in the carpal channel in the general population.

Histopathologically, in patients who have presented SS, generalized inflammation, demyelination, and axonal degeneration of the spinal nerves have been observed, although the sural nerve is usually relatively preserved, which correlates with the absence of electrophysiological alterations ${ }^{7}$. In addition, the SS can be explained based on the fact that the nerve is registered near the lateral malleolus, somewhere between the spinal roots and its distal end in the foot, where there seems to be a lower predisposition to focal demyelinating damage ${ }^{5}$.

The frequency of SS has been reported in different variants of GBS, being more frequent in the AIDP forms, followed by the MF syndrome (MFS) and occasionally found in patients with AMAN. It is not strange to observe this in the MFS that is mostly shown as a demyelinating form of GBS. However, due to the low prevalence found in SS and the high frequency of axonal forms described in our setting, it seems evident that SS is not applicable in electrophysiological screening to differentiate forms of $\mathrm{GBS}^{8}$.

\section{Conclusion}

In conjunction with the clinical picture and other typical neurophysiological findings, the preservation of the sural nerve in the GBS seems to be a diagnostic support tool, which also adds to the fact that it is easy to obtain in the practical electrodiagnostic environment. In an isolated way, it provides information on the physiopathology of the phenomenon in the classic forms of GBS, mostly demyelinating. In the Mexican population, where axonal forms prevail, it is assumed that routine SS screening should not provide diagnostic support related to the low frequency in which it has been found in this study (17.6\% of cases), so it should be considered in populations with predominance of the axonal form as complementary diagnostic method. 


\section{Con囚icts of interest}

None.

\section{Ethical disclosures}

Protection of human and animal subjects. The authors declare that no experiments were performed on humans or animals for this study.

Con $₫$ dentiality of data. The authors declare that they have followed the protocols of their work center on the publication of patient data.

Right to privacy and informed consent. The authors have obtained the written informed consent of the patients or subjects mentioned in the article. The corresponding author is in possession of this document.

\section{References}

1. van Doorn PA, Ruts $L$, Jacobs BC. Clinical features, pathogenesis, and treatment of guillain-barré syndrome. Lancet Neurol. 2008;10:939-50.

2. Hadden RD, Cornblath DR, Hughes RA, Zielasek J, Hartung HP, Toyka KV, et al. Electrophysiological classification of guillain-barré syndrome: clinical associations and outcome. Ann Neurol. 1998;44:780-8.

3. Hiew FL, Rajabally YA. Sural sparing in guillain-barré syndrome subtypes: a reappraisal with historical and recent definitions. Clin Neurophysiol. 2016;127:1683-8.

4. Al-Shekhlee A, Robinson J, Katirji B. Sensory sparing patterns and the sensory ratio in acute inflammatory demyelinating polyneuropathy. Muscle Nerve. 2007;35:246-50.

5. Umapathi T, Li Z, Verma K, Yuki N. Sural-sparing is seen in axonal as well as demyelinating forms of guillain-barré syndrome. Clin Neurophysiol. 2015;126:2376-80.

6. Grimm A, Oertl H, Auffenberg E, Schubert V, Ruschil C, Axer H, et al Differentiation between Guillain-Barré syndrome and acute-onset chronic inflammatory demyelinating polyradiculoneuritis-a prospective follow-up study using ultrasound and neurophysiological measurements. Neurotherapeutics. 2019;16:838-47.

7. Bromberg MB, Albers JW. Patterns of sensory nerve conduction abnormalities in demyelinating and axonal peripheral nerve disorders. Muscle Nerve. 1993:16:262-6.

8. Derksen A, Ritter C, Athar P, Kieseier BC, Mancias P, Hartung HP, Sheikh KA, and Lehmann HC. Sural sparing pattern discriminates Guillain-Barré syndrome from its mimics. Muscle Nerve. 2014:50:780-4. 